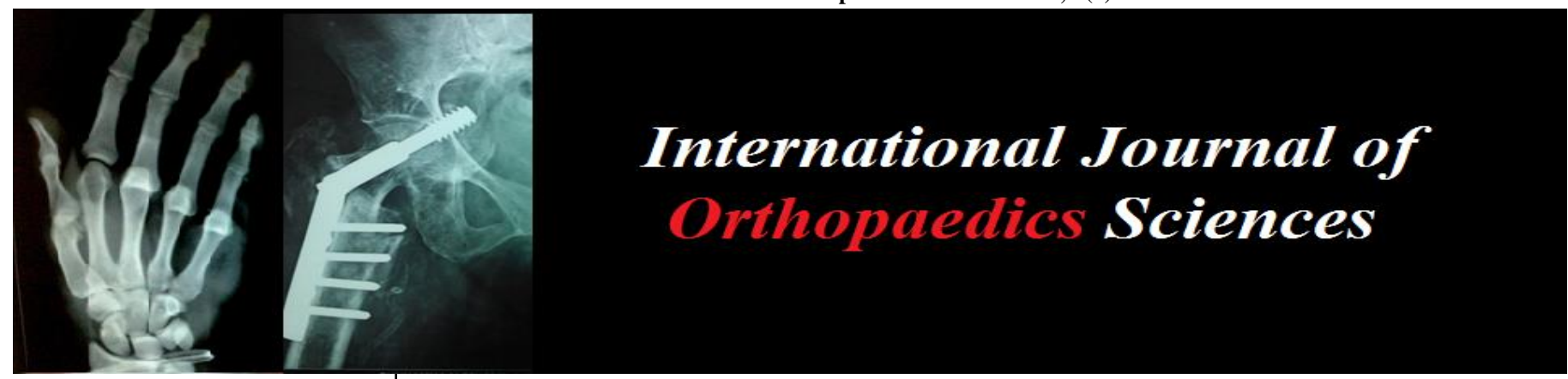

ISSN: $2395-1958$

IJOS 2018; 4(1): 940-943

(C) 2018 IJOS

www.orthopaper.com

Received: 25-11-2017

Accepted: 26-12-2017

\section{Dr. S Gopinath}

Sri Devaraj Urs Medical College,

Tamaka, Kolar, Karnataka,

India

\section{Dr. NS Gudi}

Professor in Orthopaedics, Sri

Devaraj Urs Medical College,

Tamaka, Kolar, Karnataka,

India

Dr. S Das

Professor in Pathology, Sri Devaraj Urs Medical College, Tamaka, Kolar, Karnataka, India
Correspondence

Dr. S Gopinath

Sri Devaraj Urs Medical College,

Tamaka, Kolar, Karnataka,

India

\section{A study of outcome of autolougous platelet rich plasma injection in patients with chronic plantar fasciitis}

\author{
Dr. S Gopinath, Dr. NS Gudi and Dr. S Das
}

DOI: $\underline{\text { https://doi.org/10.22271/ortho.2018.v4.i1n.135 }}$

Abstract

Plantar fasciitis is one of the most common musculoskeletal problems in the orthopaedic practice. Any heel pain due to plantar fasciitis persisting will often distress the patient, so right intervention at the right time is needed. Need of the study is to evaluate the response rate and outcome of autologous platelet rich plasma injections in patients with chronic plantar fasciitis. In studies elsewhere autologous platelet rich plasma injections reduced heel pain.

100 painful heels were screened and evaluated in this study, with base line characteristics of sex 36 were females, 26 were males. Among this 38 received bilateral PRP injections and 24 received unilateral PRP injections. The mean VAS value before injection was $7.48 \pm 0.948$ and after 3 months post injection it was $4.97 \pm 1.35$. And hence significant improvement was noted.

In our study a significant number of patients showed improvement in symptoms. There were no complications. Symptomatic and persistant relief enabled the patients to perform their daily activities and return to work. PRP injection may prove to be a superior alternative to the existing treatments for chronic heel pain.

Keywords: autologous platelet rich plasma, plantar fasciitis, visual analogue scale

\section{Introduction}

Plantar fasciitis is a common pathological condition affecting the hind foot, and can often be a challenge for clinicians to successfully treat ${ }^{[1]}$. It is an overuse injury causing inflammation at the origin of the plantar fascia and surrounding perifascial structures, such as the calcaneal periosteum. It is the most common cause of infero-medial heel pain in adults ${ }^{[2]}$. Lapidus and Guidotti ${ }^{[3]}$, in 1965 , found that the number of patients in their foot clinic with plantar fasciitis was greater than those with any other recorded foot lesion. With regard to the history of the disease, plantar fasciitis as an overuse syndrome was recognized almost two hundred years ago by an author named Davis ${ }^{[4]}$ and other authors have referred to plantar fasciitis by different names such as heel pain syndrome, subcalcaneal pain syndrome, calcaneodynia, subcalcaneal bursitis, calcaneal periostitis, neuritis, heel spur syndrome, subcalcaneal spur syndrome, stone bruise, medial arch sprain, runner's heel, jogger's heel, and policeman's heel ${ }^{[4]}$.

The different names given by different authors is due to the confusion about the etiology ${ }^{[5]}$ of plantar fasciitis many authors in their study stated that Successful treatment of plantar fasciitis usually requires a combination of treatment modalities, than administering only one treatment at a time ${ }^{[6]}$. It is stated that mechanical interventions in combination to any other treatment will relieve the heel pain to the maximum extent, but there are studies which shows that mechanical intervention is not of much significance. The mechanical treatment modalities like foot orthoses, foot taping, footwear, night splints, rest, and walking casts is stated that these will reduce the load and stress applied to the inflamed plantar fascia to a tolerable level ${ }^{7}$.Plantar fasciitis is the degenerative problem of the plantar fascia resulting from repetitive trauma at its origin on the calcaneus. Plantar fasciitis causes heel pain in both active and sedentary adults of all ages ${ }^{[8]}$.

Few authors (Kaya 2010, Chen 2010) stated that plantar fasciitis is an inflammatory disease at an acute stage due to mechanical overload will lead to chronic inflammation and degenerative changes ${ }^{[6]}$. Plantar fasciitis accounts for 11 to $15 \%$ of all foot problems in adults, it peaks 
between 40 to 60 years of age group and in younger age group like runners, the predominance of this condition according to sex varies from one study to other. (cole et al. 2005, Gill and kiebzak 1996, Neufeld and cerrato 2008) The most common site of abnormality is near the origin of plantar aponeurosis at the medial plantar tubercle of the calcaneus ${ }^{[9]}$.

Due to multifactorial ${ }^{[10]}$ causes of plantar fasciitis the treatment modalities are wide in number, to relieve pain NSAIDS, night splints, low dye taping, heel pads, cups, orthoses, steroid injection were used. Extra corporeal shock wave therapy is used in the recent years to treat this disease with life style modifications only 5 to $10 \%$ of the people will need surgical intervention like removal of calcaneal spur, neurectomy, plantar fasciotomy.

The advent of platelet rich plasma for treatment of plantar fasciitis in recent times is due to its wide advantages with early recovery of pain levels and improved functional activities of the patient in comparison with above mentioned treatments.

\section{Methods}

\section{Study Population}

A study group hundred painful heels who had heel pain persisting of 4 to 6 months with age of $18 y$ rs and above were taken up for the study.

Patients characteristics like history of heel pain, age, height, gender, weight, hours spent on standing, duration of symptoms and types of prior treatment should be noted.

Any intervention procedure will be done after taking the written consent by the patient.

\section{Inclusion Criteria}

Patients with heel pain of duration of 4 to 6 months were included in the study.

Patients with the following history were excluded:

1. Recent trauma.

2. Any radicular pain.

3. Any previous surgeries around the foot ankle.

4. Any neurological abnormalities.
5. Pregnancy

6. Low platelet count $(<1.05 / \mathrm{uL})$

\section{Preparation of Platelet Rich Plasma}

Autologous blood obtained from the patient was subjected to centrifugation methods to obtain platelet concentrate. Marx technique involving double centrifugation was used. The platelet rich plasma was assessed for platelet count, and this plasma was injected under full aseptic conditions to the painful heel.

The entire procedure is done as an office procedure ${ }^{[11]}$.

Stage 1: Anti-platelet drugs were withdrawn 10 days before the procedure. Approximately 5\% of the body weight is the estimated volume of the blood required.

Stage 2: Suitable vein is selected and blood is collected within 10 mins using standard procedure with double bag.

Stage 3: Approximately $75 \mathrm{ml}$ of blood is withdrawn after adjusting the anticoagulant in the bag with $15 \mathrm{ml}$. PRP is separated by two step configuration - $1500 \mathrm{rpm}$ for 15 minutes, then $2000 \mathrm{rpm}$ for another 15 minutes and kept in incubator for 30 to 60 minutes.

Stage 4: (Fig-1) After incubation, bag is kept on platelet agitator for at least 10 minutes before issuing. Accepted platelet rich plasma concentrate should have two times increase in platelet concentration.

The outcome study is analyzed by its pain relief and comfortable return to daily activities. Assessing the outcome is done in 2 nd week and 3rd month of the study group by visual analogue score (0-no pain, 10-maximum pain) and statistically evaluated.

Patients who did not follow the regime of treatment and who did not come for follow up were excluded and a complete description of responses to the outcome is be presented in the statistical data.

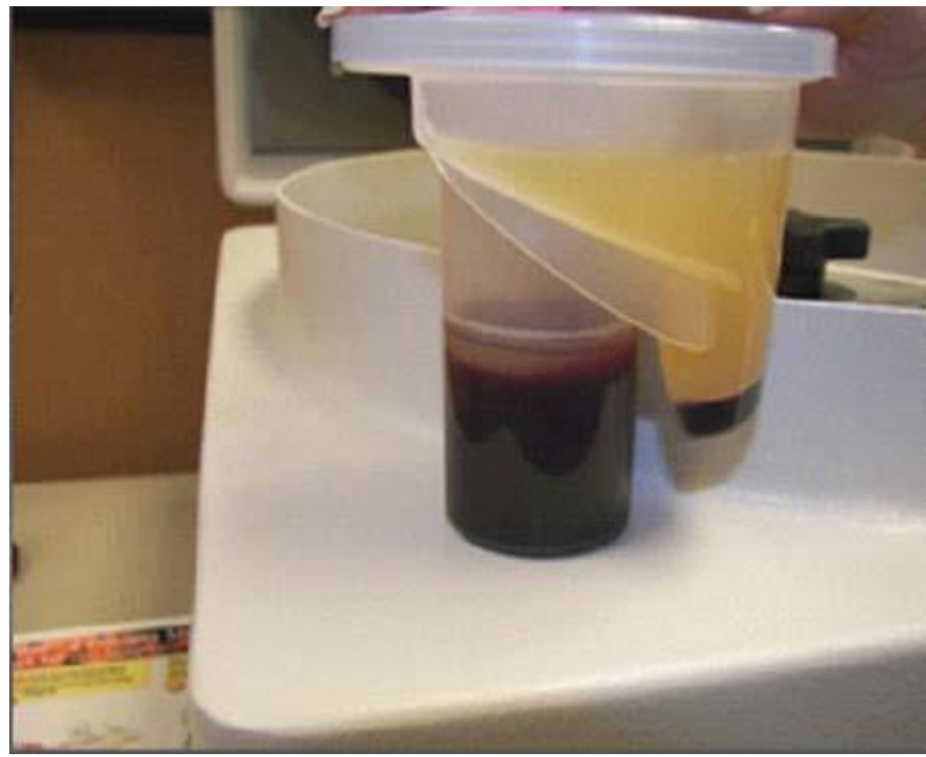

Fig 1: Red layer indicates platelet rich plasma \& yellow layer indicates platelet poor plasma ${ }^{[12]}$.

\section{Injection Technique}

The procedure was explained to the patient and consent obtained. The area to be injected was cleaned thoroughly.
22 gauge needle and $5 \mathrm{ml}$ syringe is used to draw 3 to $5 \mathrm{ml}$ of platelet rich concentrate and is injected in to the area of maximal tenderness. A single skin poke with peppering technique is used. 


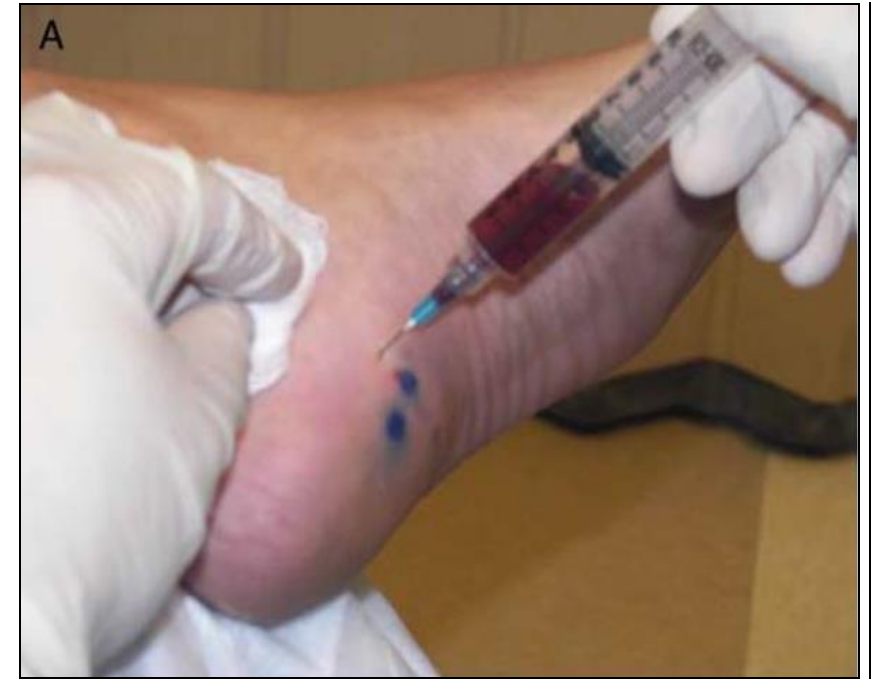

Site of injection: The Maximum Tender Point ${ }^{[12]}$.

\section{Post injection protocol}

Immediately after the injection, the patient is kept in sitting position without moving the foot for 15 minutes. Patients will go to the physiotherapist to obtain stretching exercises. Patients are sent home with instructions to limit their use of the feet for approximately 48 hours and use hydrocodone or acetaminophen for pain. The use of nonsteroidal medication is prohibited. After 48 hours, patients are given a standardized stretching protocol to follow for 2 weeks. A formal strengthening program is initiated after this stretching. At 4 weeks after the procedure, patients are allowed to proceed with normal sporting or recreational activities as tolerated. Any type of foot orthoses will not be allowed.

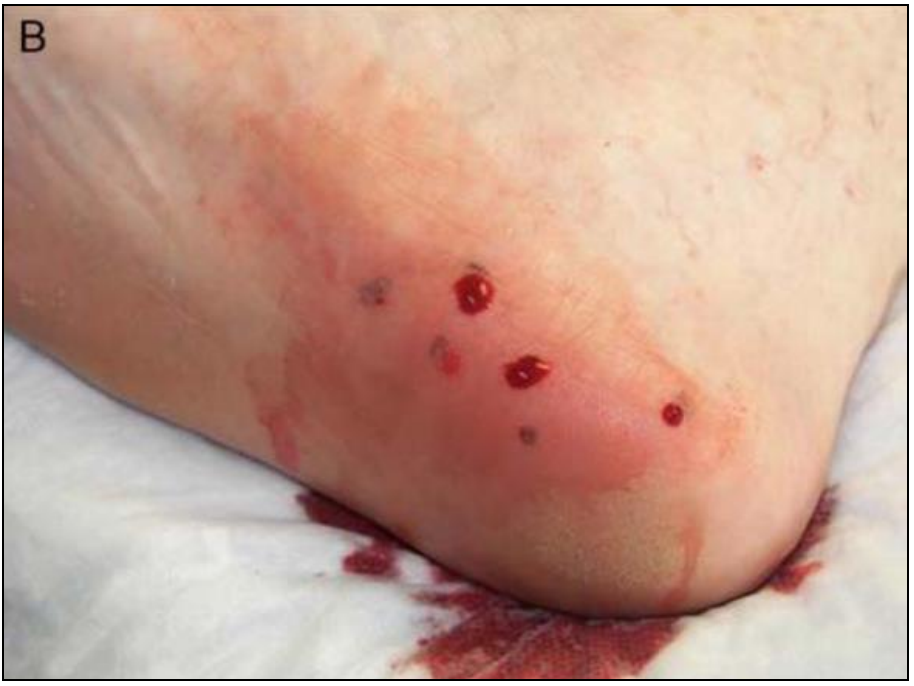

Results

Table 1: Sex distribution among the subjects

\begin{tabular}{|c|c|c|}
\hline Sex & Frequency & Percentage \\
\hline Female & 36 & 58.07 \\
\hline Male & 26 & 41.93 \\
\hline Total & 62 & \\
\hline
\end{tabular}

Table 2: No of sites used for platelet injection.

\begin{tabular}{|c|c|c|}
\hline No of sides & Frequency & Percentage \\
\hline Bilateral (both heels) & 38 & 61.29 \\
\hline Unilateral (One heel) & 24 & 38.71 \\
\hline Total & 62 & \\
\hline \multicolumn{2}{|l}{} \\
\hline
\end{tabular}

Table 3: Mean values of Age and Duration of Complaints

\begin{tabular}{|c|c|c|}
\hline & Age (Years) & Duration of Complaints (Months) \\
\hline Mean & 43.51 & 12.95 \\
\hline Std. Deviation & 12.530 & 10.132 \\
\hline
\end{tabular}

In the study the Mean age of the subjects was $43.51 \pm 12.5$ years and mean duration of complaints is $12.95 \pm 10.13$ months.

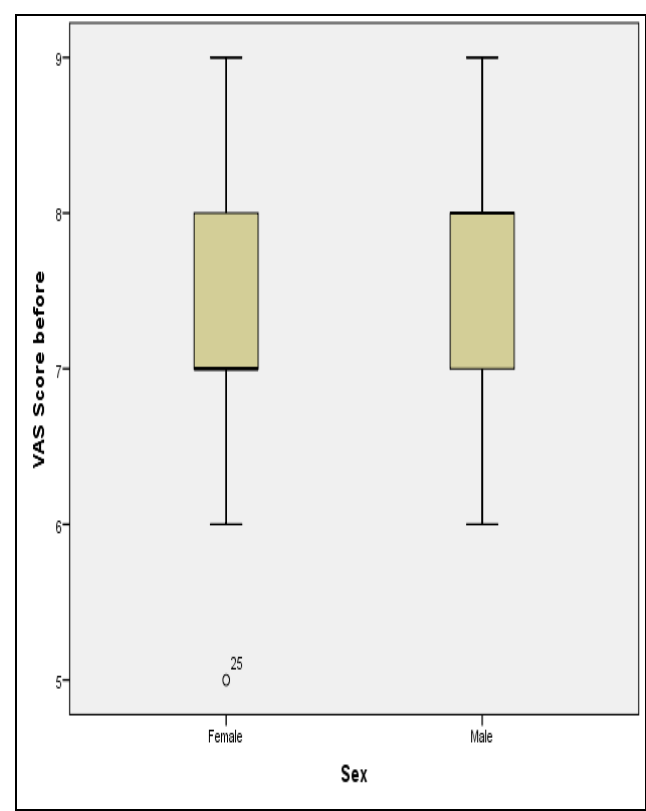

Graph 1: Box plot showing Mean scores of VAS score before injection for males and females.

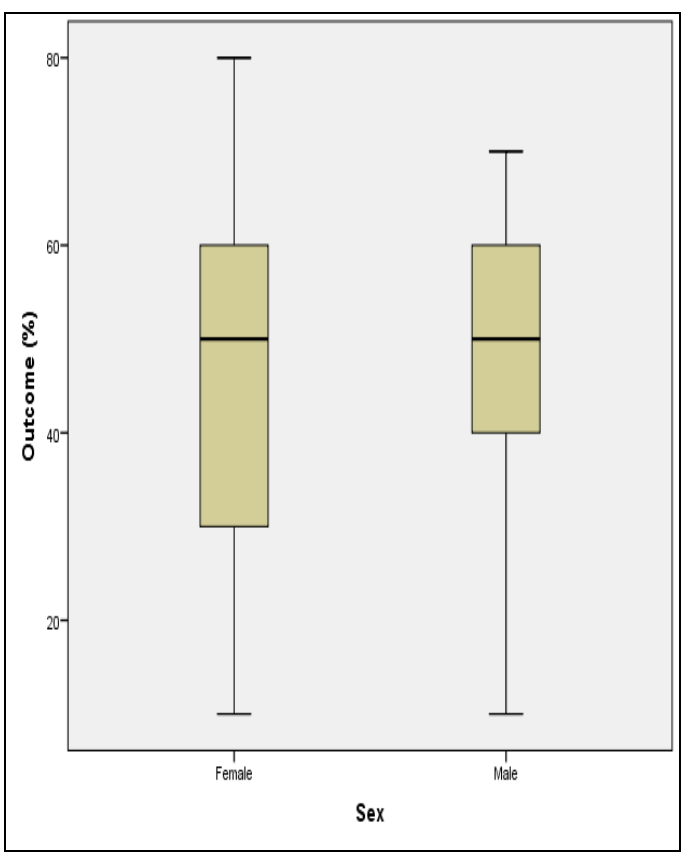

Graph 2: Box plot showing Mean scores of VAS Scores after injection for males and females at 3 months

In the study it was observed that there was significant reduction in VAS score before and after the injection. VAS 
mean score before the injection was $7.48 \pm 0.948$ and after the injection was $4.97 \pm 1.35$. The mean VAS score before the injection was high and after injection it decreased at a very significant level at 2 weeks and at 3 months.

Table 4: Showing improvement in the outcome after injection

\begin{tabular}{|c|c|c|}
\hline Outcome \% & Frequency & Percent \\
\hline 10 & 5 & 5.0 \\
\hline 20 & 10 & 10.0 \\
\hline 30 & 10 & 10.0 \\
\hline 40 & 12 & 12.0 \\
\hline 50 & 18 & 18.0 \\
\hline 60 & 31 & 31.0 \\
\hline 70 & 8 & 8.0 \\
\hline 80 & 6 & 6.0 \\
\hline Total & 100 & 100.0 \\
\hline
\end{tabular}

In the study it was observed that majority i.e. $31 \%$ had $60 \%$ improvement after the injection in VAS score, followed by $50 \%$ in $18 \%$ of individuals and highest improvement of $80 \%$ in $6 \%$ of patients and least improvement of VAS score $10 \%$ was observed among $5 \%$ of subjects.

\section{Discussion}

We designed this study to determine the outcome of platelet rich plasma injection in patients with plantar fascitis. Plantar fascitis is a very common musculoskeletal problem encountered in orthopaedic day today practice. Heel pain or plantar fascitis when acute or when chronic is quite a disabling condition. It certainly affects the day to day quality of life of patients.

Many treatment modalities have been in practice. Physiotherapy and bracing have been advised. Corticosteroid injections have been extensively used. Their efficacy is still conflicting. Corticosteroid injection can lead to local and permanent damage to structure of fascia.

With advert of biological methods of treatment in orthopaedics platelet rich plasma has been used in many clinical problems viz., wound hemostasis/healing, augmentation of bone grafts, ACL injuries and treatment of tendinosis.

In a study by Barett et al. 6 of the 9 patients achieved complete symptomatic relief after 2 months. Additional PRP injection successfully relieved the symptoms in another patient ${ }^{[11]}$. In a study by Mishra et al of 20 patients $60 \%$ showed improvement in 8 weeks, $81 \%$ at 6 months, and $93 \%$ at $1 \frac{1}{2}$ year of follow up ${ }^{[13]}$. In a study done by Ajit P.chitre of 8 patients $100 \%$ showed improvement in 3 months ${ }^{[14]}$.

We chose to study patients with plantar fascitis as it is a very common clinical problem involving the weight bearing portion of the limb.

100 painful heels were screened and evaluated in this study, with base line characteristics of sex 36 were females, 26 were males. Among this 38 received bilateral PRP inactions, 24 received unilateral PRP injections. Site of injection shows $53 \%$ right, $47 \%$ left. The mean age groups of patients are $43.51 \pm 12.5$ years and mean duration of complaints are 12.95 \pm 10.13 weeks. There was considerable improvement in visual analogue score before injection with $\mathrm{P}$ value of 0.878 to VAS score after 3 months with $P$ value 0.947 .

The mean VAS value before injection was $7.48 \pm 0.948$ and after 3 months post injection it was 4.97 \pm 1.35 . And hence significant improvement was noted.

In this study the base line characteristics like age, sex, duration of symptoms, site of symptoms does not affect the outcome of the study statistically. In this study the outcome of PRP injection is as follows.

$31 \%$ had $60 \%$ improvement in VAS score, $18 \%$ had $50 \%$ improvement in VAS score, 6\% had $80 \%$ with highest improvement in VAS score, and the least improvement is $10 \%$, was observed in $5 \%$ of subjects. These results were at the end of 3 months. Majority of the PRP treated patients had improved quality of life status. The results of the study were comparable to other short term follow up in the literature.

Limitations of this study could be short follow up and platelet concentrate was variable in nature. However PRP injection/intervention as a biological modality of treatment in orthopaedic condition is encouraging.

\section{Conclusion}

PRP is a biological treatment option for common and recalcitrant orthopaedic problems like heel pain and plantar fasciitis.

In our study, a significant number of patients showed improvement in symptoms and there were no complications. Symptomatic and persistent relief enabled the patients to perform their daily activities.

PRP injection may prove to be a superior alternative to the existing treatment options for chronic heel pain.

\section{References}

1. Cornwall MW, McPoil TG. Plantar fasciitis: Etiology and treatment. J Ortho Sports Phys Ther. 1999; 9:756-756.

2. Young CC, Rutherford DS, Niedfelt MW. Treatment of plantar fasciitis. Am Fam Physician. 2001; 63:46774:477-478.

3. Lapidus PW, Guidotti FP. Painful heel: report of 323 patients with 364 painful heels. Clinical Orthopaedics \& Related Research. 1965; 39:178-86.

4. Davies MS, Weiss GA, Saxby TS. Plantar fasciitis: how successful is surgical intervention?. Foot \& Ankle International. 1999; 20:803-7.

5. Singh D, Angel J, Bentley G, Trevino SG. Fortnightly review. Plantar fasciitis. British Medical Journal. 1997; 315:172-5.

6. Wolgin M, Cook C, Graham C, Mauldin D. Conservative treatment of plantar heel pain: long-term follow-up. Foot \& Ankle International. 1994; 15:97-102.

7. Lynch DM, Goforth WP, Martin JE, Odom RD, preece CK, Kotter MW. Conservation treatment of plantar of plantar fasciitis. A prospective study. J Am Podiatr Med Assoc. 1998; 88:375-380.

8. Kaya BK. Plantar fasciitis. J. Sport Rehabil. 1996; 5:305320.

9. Neufeld SK, Cerrato R. Plantar Fasciitis: Evaluation and treatment. J Am Acad Orthop Surg. 2008; 16:338-346.

10. Glazer JL. An approach to the diagnosis and treatment of plantar fasciitis. Foot Ankel Int. 1996; 17:57-532.

11. Barrett S, Errwdge S. Growth factors for chronic plantar fascitis. Podiatry Today. 2003; 17:37-4215.

12. Scioli M. Treatment of recalcitrant enthesopathy of the hip withplatelet-rich plasma a report of three cases. Clinical Orthopaedic Society News. Spring, 2006, 6-7.

13. Mishra A, Paelko T. Treatment of chronic elbow tendinosis with buffered platelet rich plasma. Am J Sports Med. 2006; 34(11):1774-1778.

14. Ajit Chitre P, Varsha Pancholi, Shridhar Archik. Extra Venous Use of Autologous Platelet Rich Plasma: Beginning of New Era of Therapy of Transfusion Medicine? 